\title{
L'enseignement professionnel au cœur des innovations
}

Le cas de la France

Vocational education as a focus for innovation. The case of France

La educación profesional en el centro de las innovaciones. El caso de Francia

\section{Michel Rage}

\section{OpenEdition}

\section{Journals}

Édition électronique

URL : https://journals.openedition.org/ries/4624

DOI : $10.4000 /$ ries 4624

ISSN : 2261-4265

\section{Éditeur}

France Education international

\section{Édition imprimée}

Date de publication : 1 avril 2016

Pagination : 85-95

ISBN : 978-2-85420-610-4

ISSN : 1254-4590

\section{Référence électronique}

Michel Rage, «L'enseignement professionnel au cœur des innovations », Revue internationale d'éducation de Sèvres [En ligne], 71 | avril 2016, mis en ligne le 01 avril 2018, consulté le 02 juillet 2021. URL : http://journals.openedition.org/ries/4624 ; DOI : https://doi.org/10.4000/ries.4624 


\title{
L'enseignement professionnel au cœur des innovations
}

\section{Le cas de la France}

\author{
Michel Rage \\ Inspection générale de l'éducation nationale
}

Le modèle français d'enseignement technique apparaît à plus d'un titre original par rapport à la plupart des systèmes étrangers. Trois particularismes nationaux ont contribué, chacun à leur manière, à en former l'image.

1) La rupture introduite dans le système de formation corporatiste par le décret d'Allarde et la loi Le Chapelier (1791) : en rupture avec l'ancien système d'apprentissage proche des métiers, elle permet le développement d'un enseignement scolarisé des techniques, puis des technologies ce qui rend la situation française sensiblement différente du système allemand.

2) La difficulté à dépasser, sur le plan culturel, les représentations négatives associées au monde du travail et de la production. La vieille distinction entre les arts libéraux et les arts mécaniques est toujours présente. La définition de « mécanique » dans l'édition de 1865 du dictionnaire de conversation comme «adjectif désignant les professions qui semblent demander plus au bras qu'à l'intelligence » marque aujourd'hui comme hier l'image de l'enseignement technique et surtout professionnel et constitue, en France, un handicap à son développement.

3) L'ambivalence des milieux professionnels, qui revendiquent la responsabilité de la formation professionnelle, tout en se montrant rapidement très réticents à la prendre en charge dans sa totalité et dont la culture de l'apprentissage reste très limitée.

Ces particularités rendent compte des péripéties de l'histoire de l'enseignement technique. Depuis quelques décennies, on ne peut plus parler d'un enseignement technique autonome mais plutôt de voies et filières du système éducatif qui préparent leurs élèves à des diplômes technologiques ou professionnels. Elles restent néanmoins mal connues et font parfois l'objet de critiques tant internes qu'externes malgré les richesses dont elles sont porteuses, en particulier sur le plan pédagogique : intégration interactive des aspects théoriques et pratiques de la formation, implication des élèves dans des activités de projet, relations diversifiées et enrichissantes avec les milieux professionnels.

L'histoire nous rappelle qu'un système d'enseignement est toujours le fruit des débats, des contingences, des volontarismes et des peurs de son époque, et ne saurait donc être figé. Si les mêmes débats ressurgissent, c'est sans doute 
que les équilibres entre professionnalisation et formation générale requièrent des arbitrages, des compromis variables selon les époques.

Les divers arguments avancés depuis la naissance de la formation professionnelle, par les principaux protagonistes en présence pour instaurer des certifications ayant une valeur nationale ou, au contraire, pour maintenir et développer des certifications spécialisées à telles ou telles branches économiques, montrent la difficulté à imposer une certification unique validant l'acquisition de compétences.

Jusqu'au début du XIX ${ }^{\mathrm{e}}$ siècle, le seul modèle existant était celui du compagnonnage. Dès 1880 et la création des écoles manuelles d'apprentissage, l'éducation et l'industrie s'opposent sur la tutelle de l'enseignement professionnel et technique. La problématique est longtemps - toujours ? - restée la même : une formation dont les contenus permettent une opérationnalité immédiatement ou une formation incluant plus d'enseignements généraux pour accroître le potentiel d'évolution.

C'est après la Deuxième Guerre mondiale que se met en place la structure actuelle par niveaux de formation, le technique court (CAP, BEP), le technique long (baccalauréats technique et des sciences et techniques) et les écoles d'ingénieur. Les trente années d'après guerre ont été marquées par une croissance économique exceptionnelle permettant aux enseignements technique et professionnel de constituer des voies d'accès aux études prolongées pour les jeunes majoritairement issus des classes populaires. Ces enseignements ont permis, durant cette période, une mobilité sociale importante par les promotions de carrière. L'intégration de la formation professionnelle dans l'appareil scolaire et la massification d'une poursuite d'étude au sein de la voie générale érigée en politique, en fixant l'objectif de $80 \%$ d'une classe d'âge au niveau du baccalauréat, ont mis fin à cet âge d'or. La création du baccalauréat professionnel, au début des années 1980, est une réponse à une demande de l'Union des industries métallurgiques et minières (UIMM), qui s'inscrit dans le cadre d'une politique scolaire instituant le baccalauréat en norme, appliquée à tous les secteurs professionnels. Cette création marque le développement d'une réflexion influençant encore aujourd'hui la structuration des filières technologiques et professionnelles.

Historiquement les premières certifications adoptées concernent un petit nombre de formations : celles proposées par les écoles pratiques de commerce et d'industrie (EPCI) et les écoles nationales professionnelles (ENP), qui deviendront des lycées techniques, sont censées former des ouvriers instruits, aptes à devenir contremaîtres et chefs d'atelier. Mais leur généralisation à l'apprentissage d'un métier reste controversée jusqu'en 1919. C'est en effet à cette date que la loi Astier institue le certificat d'aptitude professionnelle (CAP). Ce dernier succède au certificat de capacité professionnelle (créé en 1911), qui devait permettre de reconnaître les connaissances pratiques, théoriques et techniques possédées par ses titulaires. Mais si le diplôme est finalement retenu comme 
mode de certification des formations professionnelles avant la Seconde Guerre mondiale, les modalités de sa délivrance, l'organisation des examens ont continué à dépendre des contingences locales jusqu'à la fin des années 1930. C'est donc à la faveur d'une conjoncture politique qui renforce le pouvoir de l'État républicain et d'une évolution du marché du travail que la délivrance des diplômes et leur organisation sont devenues le monopole de l'État. Désormais, les représentants des organisations professionnelles patronales sont seulement associés à leur élaboration et à celle des examens qui leur sont attachés. L'adoption des diplômes nationaux, sorte de mesure de la valeur d'échange sur le marché du travail, ne s'accompagne pas d'une disparition des certifications professionnelles, mais celles-ci ne sont généralement que des spécifications de ceux-là.

C'est à la Libération que se mettent en place les bases de l'organisation actuelle des formations professionnelles scolarisées. Les centres d'apprentissages, futurs collèges des enseignements techniques (CET), puis lycées d'enseignement professionnel (LEP) et enfin lycées professionnels (LP) sont destinés à la formation des ouvriers et se posent en concurrents directs de l'apprentissage. Les collèges techniques et les écoles nationales professionnelles (ENP) sont exclusivement consacrés à la formation de techniciens, annonçant leur transformation en lycées techniques en 1959. Parallèlement l'AFPA ${ }^{1}$ est organisée dès 1945 et vise plus particulièrement la formation d'adultes.

\section{Commissions PROFESSIONNELLES CONSULTATIVES ET DIPLÔMES GARANTIS PAR L'ÉTAT}

De 1945 à 1985, en accompagnant la massification de la scolarité obligatoire, l'enseignement professionnel a connu un accroissement ininterrompu de ses effectifs jusqu'à scolariser $30 \%$ des lycéens, l'enseignement technique progressant également mais de manière moins spectaculaire. Afin de réglementer les formations professionnelles et de standardiser leur mode de certification à l'échelle nationale, l'État crée en 1948 les commissions nationales professionnelles consultatives, qui deviendront les commissions professionnelles consultatives (CPC) actuelles. Structurées par branches d'activités, les CPC peuvent modifier, créer ou supprimer des diplômes en fonction des besoins manifestés par les représentants des professions.

On compte de nos jours quatorze CPC relevant du ministère de l'éducation nationale, cinq du ministère de l'emploi et de la solidarité, une du ministère des affaires sociales, du travail et de la solidarité, une du ministère de la jeunesse et des

1. L'Association nationale pour la formation professionnelle des adultes (Afpa) est un organisme français de formation professionnelle au service des régions, de l'État, des branches professionnelles et des entreprises. Elle propose des formations qualifiantes sanctionnées par un titre professionnel du ministère du travail. 
sports et une relevant de l'agriculture. Elles sont amenées à définir des profils d'emplois, des contenus de formations et des règlements d'examens. Elles sont le lieu de concertation entre le ministère (direction générale de l'enseignement scolaire et inspection générale pour l'éducation nationale), le ministère du travail, les représentants des organisations patronales et ouvrières, des syndicats d'enseignants et des parents d'élèves. Les diplômes négociés dans les CPC, reconnus dans les conventions collectives et garantis par l'État, jouent le rôle de véritables instruments de mesure de la qualification. Les diplômes professionnels de l'éducation nationale sont automatiquement inscrits au répertoire national des certifications professionnelles (RNCP) du fait de l'existence des CPC. Le référentiel des activités professionnelles (RAP), présent dans tous les diplômes, garantit la légitimité des diplômes professionnels auprès des employeurs et des salariés français, en déclinant les tâches couvertes par les activités visées par le diplôme ; constitue un langage commun pour les échanges entre tous les acteurs de la formation et de la certification professionnelle; fournit une référence concrète pour l'évaluation des compétences.

C'est donc à partir des activités professionnelles correspondant à des processus de travail, décomposées en tâches (ensemble d'opérations élémentaires mises en œuvre pour réaliser le travail prescrit) que nous pouvons établir des relations avec les compétences ${ }^{2}$ professionnelles.

\section{DiPLÔMES, TITRES ET CERTIFICATIONS}

Le paysage de la formation professionnelle a été bouleversé dans les années 1980. Les entreprises adoptent de nouvelles organisations du travail et de gestion de la main d'œuvre rompant avec l'organisation «taylorienne ». On passe ainsi d'une logique "un homme, une machine" à " un système, une équipe " et les premières révolutions techniques dues au numérique font leur apparition. L'État crée alors un nouveau diplôme, le baccalauréat professionnel donnant naissance à un nouveau type d'ouvrier qualifié dont les entreprises attendent une maîtrise élargie des compétences techniques, une aptitude à travailler en équipe et une véritable culture industrielle. Il faut insister sur la profonde mutation qu'ont subie les lycées professionnels, par l'élévation du niveau des qualifications d'une part, et de l'autre par les exigences d'apport de culture générale dues au choix stratégique d'un baccalauréat et non d'un quelconque diplôme professionnel. Les enseignants se sont formés, ont su intégrer les évolutions techniques et ont conservé les pratiques pédagogiques. Cette élévation du niveau de qualification conjugué à l'objectif proclamé de $80 \%$ d'une

2. L'Association française de normalisation (AFNOR) a proposé une normalisation de la mesure des compétences souscrivant, en les approfondissant, aux préconisations du fascicule de documentation FD $X_{50-183}$. Cette norme est complétée par les définitions suivantes : « la compétence est "une capacité éprouvée à mettre en œuvre des connaissances, des savoir-faire et comportements en situation d'exécution. C'est la capacité éprouvée à résoudre des problèmes dans un contexte donné" " . 
classe d'âge au niveau du baccalauréat a contribué à la modification profonde de l'offre de formation professionnelle. Les CAP ont été progressivement abandonnés au profit d'un cursus BEP - baccalauréat professionnel. Le taux d'accès dans les formations CAP passe ainsi de $15 \%$ d'une classe d'âge en 1981 à 1,5\% en 1991. Le CAP perd son identité de premier diplôme qualifiant, se prépare principalement par apprentissage et devient, excepté pour l'artisanat et dans quelques spécialités porteuses d'emploi à ce niveau (électricité, restauration par exemple), réservé à certaines catégories d'activités peu valorisantes ou destiné à offrir une formation minimale aux exclus de l'école.

\section{Graphique 1. Filière chaudronnerie : évolution des flux entrants et sortants de 1997 à 2013 pour la voie scolaire}

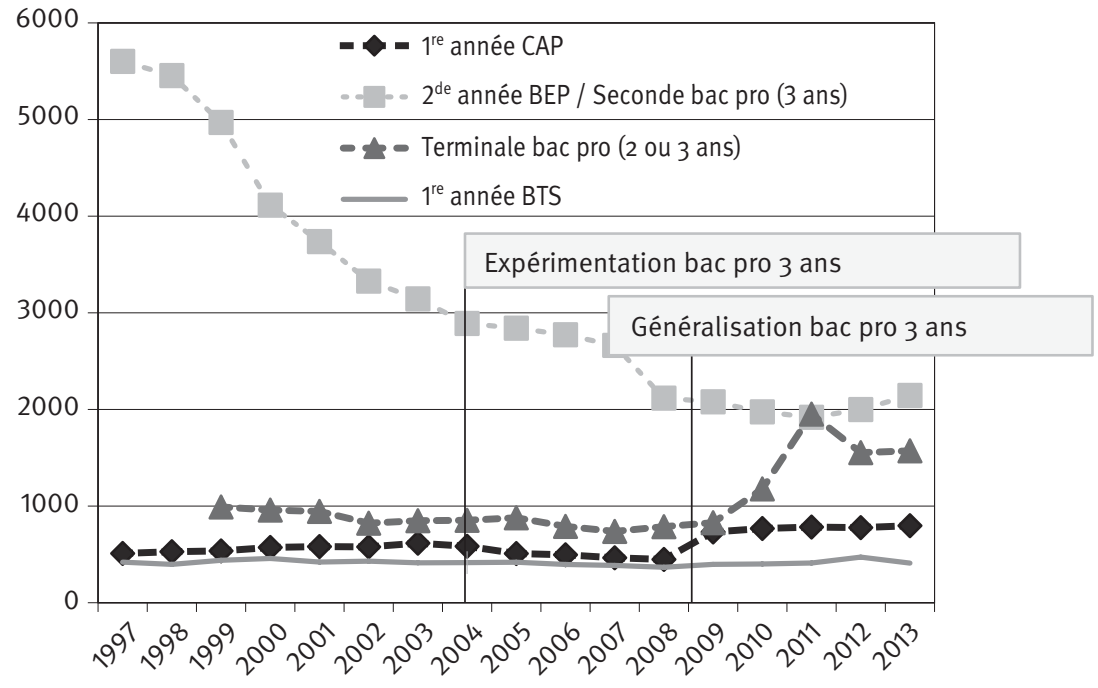

La chute des effectifs de BEP est spectaculaire mais l'effet du passage en trois ans du baccalauréat professionnel a réussi à inverser la courbe et permis le redressement des CAP, dorénavant seuls diplômes de niveau V et qui représentent en moyenne $25 \%$ des entrants post troisième en lycée professionnel. Les effectifs en BTS sont stables, grâce à la présence de plus en plus importante des baccalauréats professionnels (en moyenne $25 \%$ du flux d'entrée, tous BTS confondus mais avec de fortes variations selon le champ d'activités).

D’une façon générale, au niveau individuel, l'obtention d'un diplôme demeure « rentable » en termes d'insertion sur le marché du travail, même si son rendement relatif s'est érodé depuis vingt ans. Les constats sont structurels et constants depuis deux décennies : le niveau de diplôme des individus est le facteur prépondérant d'insertion professionnelle et de positionnement au sein du marché du travail. Plus le niveau de formation augmente, plus les difficultés d'insertion professionnelle décroissent. Diverses études de l'Insee et du Céreq le montrent, les jeunes diplômés restent moins longtemps au chômage ; ils se placent à de meilleurs postes et sont globalement mieux rémunérés que les non-diplômés. 
Un titre professionnel est une certification professionnelle délivrée par le ministère chargé de l'emploi. Il en existe environ 260, tous enregistrés dans le Répertoire national des certifications professionnelles (RNCP). Un titre professionnel est constitué d'une ou plusieurs unités représentant chacune un ensemble cohérent de compétences, aptitudes et connaissances : les certificats de compétences professionnelles (CCP).

Le compromis établi au lendemain de la Seconde Guerre mondiale, qui met le diplôme en adéquation avec la formation et l'associe à la détermination des grilles de classification dans les conventions collectives, est toujours resté l'objet de discussions et de tentatives d'infléchissements de la part des milieux professionnels, qui ont développé leurs propres certifications.

Depuis l'accord interprofessionnel du $1^{\mathrm{er}}$ mars 1989, les commissions paritaires nationales de l'emploi (CPNE) des branches professionnelles peuvent mettre en œuvre de véritables politiques de formation initiale et continue (Ilardi et al., 2015), produire leur propre normes de certification et délivrer des certificats de qualification professionnelle (CQP). Ce nouveau système de certification s'est largement développé et s'adresse principalement aux salariés d'entreprise mais aussi aux jeunes en situation de chômage ou de perfectionnement. Les CQP dispensent avant tout des savoirs directement opérationnels dans l'activité visée et minimisent fortement les connaissances générales. Ceci se traduit également dans les pratiques d'évaluation, qui privilégient les savoir-faire professionnels.

Les CQP constituent donc une rupture avec le monopole de la délivrance des diplômes professionnels par l'État, ces derniers n'étant plus, de fait, reconnus comme le seul débouché légitime de la formation.

L'accord national interprofessionnel de décembre 2013 tend à élargir encore le rôle des branches professionnelles, qui disposent de trois instances impliquées dans la politique emploi-formation. La commission paritaire nationale de l'emploi et de la formation professionnelle (qui succède à la CPNE), organe politique paritaire de la branche, est chargée de structurer l'offre de formation et de favoriser le développement des compétences des salariés. L'observatoire des métiers des qualifications et des compétences (OPMQC), institutionnalisé depuis l'accord national interprofessionnel (ANI) de 2003 produit les données de la branche pour piloter, orienter et informer. L'OPCA (organisme paritaire collecteur agréé), récemment réformé, met en œuvre la politique de la branche en fournissant aux entreprises un appui en matière d'ingénierie et de financement de la formation, grâce à la collecte de la taxe d'apprentissage.

La comparaison met à la fois en évidence la relative proximité entre les titres et les CQP, mais également leurs différences avec un diplôme. En effet, le diplôme intègre une importante proportion d'enseignements généraux. Pour ce qui concerne les enseignements professionnels, les situations professionnelles sont également plus larges pour le diplôme pour lequel le RAP est basé sur l'emploi que pour les titres et les CQP qui correspondent à des qualifications plus précises. 


\section{L'ALTERNANCE \\ DANS LES FORMATIONS \\ PROFESSIONNELLES}

La mise en place d'un ensemble de pratiques et de dispositifs divers, à partir des années 1970, désigné sous le nom d'alternance est l'un des changements majeurs intervenus dans les modes de formation. Ce terme représente la recherche d'une coopération entre les institutions de formation (publiques et privées) et les entreprises. Le fait d'alterner de façon formative, et non par simple juxtaposition, des périodes d'études et de travail, se heurte à de multiples oppositions structurant les esprits et les institutions entre théorie et pratique, savoirs formalisés et savoir-faire, logique de formation et logique de production... Former en alternance, c'est ouvrir le champ éducatif à de nouveaux acteurs, avec lesquels il faut négocier, contractualiser, redéfinir des rôles professionnels, en partenariat.

Si l'on admet que l'apprenant apprend par son action de travail - principe de base de la validation des acquis de l'expérience -, c'est passer d'une logique de formation par consommation de savoirs à une logique de formation par production de savoirs. Mais il faut aussi exploiter, d'un point de vue didactique, l'expérience acquise en situation de travail. Ceci suppose que les enseignants acceptent d'inverser en partie leur logique disciplinaire pour entrer dans une logique interdisciplinaire de production de savoir, à partir de l'explicitation et de la problématisation des situations vécues. L'alternance permet d'acquérir ce qui ne s'apprend pas à l'école et qui, pourtant, constitue l'essentiel de la compétence : l'expérience du travail réel. Le rapport théorie-pratique est aussi fait de réappropriation et d'investissement.

L'alternance sous statut scolaire s'affirme en 1985, avec la création des périodes de formation en milieu professionnel pour les baccalauréats professionnels, et s'étend en 1992, par la généralisation des stages en entreprise, à toutes les formations et en particulier à des diplômes de l'enseignement technique et professionnel. Les périodes de formation en milieu professionnel se distinguent des périodes de stage en ce qu'elles permettent non seulement l'application de compétences et de connaissances acquises en établissement de formation mais également l'acquisition de compétences et de connaissances qui ne peuvent être mises en ouvre que dans des situations réelles de production.

C'est cette raison qui a prévalu lorsque les périodes de formation en milieu professionnel ont été créées. Elles apparaissaient en effet comme la garantie de la professionnalité des titulaires du diplôme et, en conséquence, comme un atout pour leur insertion dans la vie active, dans un pays où l'expérience professionnelle est très souvent une condition nécessaire à l'embauche. C'est pour afficher et conforter ce rôle qu'elles ont dès l'origine fait l'objet d'une évaluation certificative dans le cadre d'une épreuve spécifique de l'examen, à laquelle sont associés les représentants de l'entreprise ou de la structure d'accueil. 
L'introduction des périodes de formation en milieu professionnel n'a pas donné lieu à une réelle prise en compte d'une pédagogie de l'alternance. Mais ceci a été sans conséquence tant que les durées de formation ont permis une redondance des enseignements et des activités professionnelles. Depuis 2009, avec la généralisation des baccalauréats professionnels en trois ans, c'est à une rupture des pratiques pédagogiques et organisationnelles à laquelle la voie professionnelle doit faire face. Cette évolution a été beaucoup plus délicate et peut-être moins perçue et aussi, moins bien accompagnée. À l'aspect "temps disponible de formation » s'ajoutent d'autres éléments comme l'hétérogénéité des élèves, l'individualisation (qu'il faudrait réaliser pour conjuguer des aspirations différentes des jeunes en classe de terminale entre emploi et poursuite d'études), le rajeunissement des élèves, les modalités de certifications (avec le contrôle en cours de formation, traduit de manière très disparate par les disciplines et de façon très chronophage) et enfin des jeunes qui contestent de plus en plus le rôle traditionnel d'un professeur uniquement dispensateur de savoirs.

L'apprentissage, qui représente la plus ancienne forme d'alternance, est rarement évoqué comme un mode typique de formation et fait actuellement l'objet d'interventions répétées de l'État pour son développement. En déclin rapide dans les années 1960, il connait un essor significatif ensuite pour atteindre 340000 apprentis en 2000, de sorte que $25 \%$ des CAP, BEP et baccalauréats professionnels sont préparés en apprentissage. Ce mode de formation s'est depuis fortement étendu à d'autres catégories professionnelles que celles consacrées par la tradition des métiers et on constate un accroissement des formations en BTS, licences professionnelles et écoles d'ingénieurs. En 2015, on comptabilise environ 400000 apprentis (chiffre encore éloigné de l'objectif de 500000 apprentis en 2017) et environ 265000 nouveaux contrats signés, en baisse par rapport aux années précédentes.

\section{LES DONNÉES DE LA FORMATION PROFESSIONNELLE LIÉES À L'INSERTION}

Le Céreq ${ }^{3}$ et l'INSEE ${ }^{4}$ sont deux organismes qui proposent de nombreuses enquêtes sur les sorties de l'appareil éducatif et l'entrée au travail. À la fin des années quatre-vingt-dix, le Céreq a mis en place un dispositif original d'enquêtes qui permet d'étudier l'accès à l'emploi des jeunes à l'issue de leur formation initiale. Tous les trois ans, une nouvelle enquête est réalisée auprès de jeunes qui ont en commun d'être sortis du système éducatif la même année, quel que soit le niveau ou le domaine de formation atteints, d'où la notion de "génération ". L'enquête permet de reconstituer et d'analyser les parcours des jeunes au cours de leurs trois premières années de vie active, au regard notamment du parcours

3. Céreq : Centre d'étude et de recherche sur les qualifications. (NdIR)

4. INSEE : Institut national de la statistique et des études économiques. (NdIR) 
scolaire et des diplômes obtenus. Certaines cohortes sont interrogées plusieurs fois pour suivre les débuts de carrière. Selon le compte rendu de la dernière enquête en date, "génération 2010 ",

"les jeunes titulaires de CAP et BEP, et, dans une moindre mesure, les bacheliers professionnels, rencontrent en 2013 les plus grandes difficultés sur le marché du travail. D'abord berceau de la «scolarisation des apprentissages» au niveau V, puis vecteur d'accès au niveau du baccalauréat pour un nombre croissant de jeunes d'origine populaire, l'enseignement secondaire peut sembler aujourd'hui perdre de son importance au sein de la société. Mais l'accent mis sur le développement de l'enseignement supérieur et l'attention portée aux décrocheurs ne doivent pas faire oublier qu'un tiers des jeunes entrent sur le marché du travail avec pour bagage un diplôme de l'enseignement professionnel secondaire.

Pour la génération sortie du système éducatif en 2010 comme pour les précédentes, mieux vaut toujours intégrer le marché du travail avec un diplôme de premier niveau que sans aucun diplôme du tout. Toutefois, les diplômés de CAP-BEP voient leur désavantage s'accroître par rapport aux bacheliers professionnels. Cet effet global est cependant à nuancer selon les spécialités de formation. (Ilardi, Sulzer, 2015)

Les enquêtes «Insertion dans la vie active " (IVA) et «Insertion professionnelle des apprentis » (IPA) rendent compte de la première insertion des sortants des formations professionnelles de lycée, sept mois après la fin de leur formation initiale. Cette enquête est réalisée par les académies et les lycées publics et privés sous tutelle du ministère de l'éducation nationale, de l'enseignement supérieur et de la recherche, quelquefois en partenariat avec les instances régionales. Elles mettent en valeur une insertion facilitée lorsque le diplôme est obtenu par apprentissage.

L'INSEE publie périodiquement, depuis 1970, en collaboration avec les ministères de l'éducation, du travail et le Céreq, les bilans formation/emploi qui renseignent sur l'insertion professionnelle des jeunes au sein des mouvements qui affectent l'emploi. L'angle d'approche adopté permet de mettre en évidence d'autres phénomènes que ceux décrits par les études du Céreq. C'est ainsi qu'apparaissent certaines données structurelles caractéristiques des dernières décennies : la part des jeunes dans l'ensemble des recrutements reste stable, quel que soit le volume des recrutements.

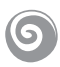

C'est un regard global qu'il est nécessaire de porter sur les systèmes de formation en France, dans un contexte politique lié à la décentralisation de la voie professionnelle. Il s'agit d'avoir pour ambition d'assurer la satisfaction des besoins des personnes et des territoires, dans une double perspective de formation tout au long de la vie et de création d'une offre territoriale cohérente de service 
et de formation. Cette exigence nécessite la construction de réponses intégrées et concertées sur un même territoire. Complexité, confusion et cloisonnement caractérisent le secteur de la formation professionnelle ; des attitudes trop idéologiques entraînent obligatoirement une perte de fonctionnalité, d'efficacité et une augmentation inévitable des coûts de structures et de moyens humains. La finalité première des diplômes professionnels est l'insertion immédiate sur le marché du travail mais la demande sociale, qui vise aussi à la reconnaissance de l'égale dignité des voies de formation, cherche à développer les possibilités de poursuite d'études, notamment en section de technicien supérieur. Cette possibilité est indispensable dans les secteurs pour lesquels le niveau d'accès à l'emploi s'est accru. Depuis 2005, tous les élèves ou apprentis qui ont obtenu une mention bien ou très bien au baccalauréat professionnel sont admis de droit dans une section de technicien supérieur (STS) et quelques classes préparatoires aux études supérieures (CPES) se sont ouvertes dans certaines régions. La réussite de ces jeunes est étroitement liée aux modalités d'accueil et à la qualité de leur préparation en amont.

L'une des missions primordiales de l'établissement de formation est d'offrir à tout jeune une solide formation générale de base autorisant l'accès à une vie professionnelle et sociale de qualité. Ainsi, l'établissement doit assurer le développement des capacités de travail personnel, de raisonnement, de jugement, de communication, de travail en équipe et de prise de responsabilités. La réforme de la formation professionnelle est déjà un succès pour au moins un objectif, celui de l'élévation du niveau de qualification des jeunes, puisque la part des bacheliers professionnels dans une génération est dorénavant d'environ $20 \%$, en augmentation par rapport à 2010. Parallèlement, le CAP attire un nombre croissant d'élèves, ce qui permet d'être optimiste sur l'atteinte du deuxième objectif : la baisse de sorties sans qualification. Le lycée polyvalent, apparu à la suite de la réforme des lycées de 1992, est l'un des outils politiques de recomposition de l'offre de formation dans une académie, par la mixité sociale des apprenants qu'il propose. La proximité immédiate des différentes voies de formation est un gage de meilleure gestion des parcours de formation, de passerelles et donc de réussite pour les jeunes. C'est aussi un signe fort de l'inscription de la formation professionnelle dans l'environnement scolaire. La France a la chance de disposer d'un outil de formation performant et prospectif. Les régions se sont engagées dans des actions de rénovation des locaux et de modernisation des équipements qui offrent aux jeunes des lieux d'apprentissage performants et de très grande qualité. Cet outil exceptionnel, qui fait la convoitise de nombreux pays étrangers, est trop souvent ignoré ou méprisé. Il mérite, comme les démarches pédagogiques innovantes développées par le corps enseignant, d'être mis en valeur et mieux utilisé. 


\section{BibLIOGRAPHIE}

Association française de normalisation, AFNOR (2002): Outils de management: Ressources humaines dans un système de management de la qualité. Management des compétences. FDX50-83, juillet, Saint-Denis La Plaine : AFNOR

AGULHON C. (1994): L'enseignement professionnel, quel avenir pour les jeunes?, Éditions de l'atelier, Paris.

D'AGOSTINO A., DIF-PRADALIER M., QUINTERO N. (2015) : "L'appui des branches professionnelles aux entreprises : trois logiques d'action ", Bref du Céreq $n^{\circ} 338$, septembre.

ILARDI V., SULZER E. (2015) : «CAP-BEP: des difficultés d'insertion encore aggravées par la crise »Bref du Céreq, ${ }^{\circ} 335$, mai.

Formation Emploi (2015), «Le bac pro a 30 ans », $\mathrm{n}^{\circ}$ 131, Paris : La Documentation française.

Formation emploi (2007): "Les usages sociaux de la compétence ", n 99, Paris : La Documentation française.

PELPEL P. et TROGER V. (1993): Histoire de l'enseignement technique, Paris : Hachette.

TANGUY L. (1991) : L'enseignement professionnel. Des ouvriers aux techniciens, Paris : PUF.

TANGUY L. (2000) : " Histoire et sociologie de l'enseignement technique et professionnel en France : un siècle en perspective ", Revue française de pédagogie, vol. 13, $\mathrm{n}^{\circ} 1$, p. $97-127$. 